\title{
Generalized superconductors from the coupling of a scalar field to the Einstein tensor and their refractive index in massive gravity
}

\author{
Manman Sun ${ }^{1}$, Dong Wang ${ }^{1}$, Qiyuan Pan ${ }^{1,2, a}$, Jiliang Jing ${ }^{1,2, b}$ \\ ${ }^{1}$ Key Laboratory of Low Dimensional Quantum Structures and Quantum Control of Ministry of Education, Department of Physics, Synergetic \\ Innovation Center for Quantum Effects and Applications, Hunan Normal University, Changsha 410081, Hunan, China \\ ${ }^{2}$ Center for Gravitation and Cosmology, College of Physical Science and Technology, Yangzhou University, Yangzhou 225009, China
}

Received: 30 August 2018 / Accepted: 31 January 2019 / Published online: 19 February 2019

(C) The Author(s) 2019

\begin{abstract}
We construct the generalized superconductors from the coupling of a scalar field to the Einstein tensor in the massive gravity and investigate their negative refraction in the probe limit. We observe that the larger graviton mass and Einstein tensor coupling parameters both hinder the formation of the condensation, but the larger graviton mass or smaller coupling parameter makes it easier for the emergence of the Cave of Winds. Furthermore, we see that the larger graviton mass but smaller coupling parameter make the range of frequencies or the range of temperatures larger for which a negative Depine-Lakhtakia index occurs, which indicates that the graviton mass and Einstein tensor have completely different effects on the negative refraction. In addition, we find that the larger graviton mass and coupling parameters both can reduce the dissipation and improve the propagation in the holographic setup.
\end{abstract}

\section{Introduction}

It is well known that Maldacena conjectured a remarkable connection between a strongly coupled field theories to a gravitational theory in higher spacetime dimensions in 1998 $[1,2]$, which states that a $d$-dimensional weakly coupled dual gravitational description in the bulk is equivalent to a $(d-1)$ dimensional strongly coupled conformal field theory on the boundary. This is the so-called anti-de Sitter/conformal field theories (AdS/CFT) correspondence [3,4], which provides a novel approach to studying the strongly coupled systems in condensed matter physics in the past ten years, especially the high temperature superconductors [5]. With this holographic duality, it is shown that the holographic superconductor models exhibit many characteristic properties shared

\footnotetext{
a e-mail: panqiyuan@126.com

b e-mail: jljing@ @unnu.edu.cn
}

by real superconductors [6]; for reviews, see Refs. [7-10] and references therein. However, in most cases, the studies on the holographic superconductors focus on the superconductors without an impurity. Turning on a coupling between the gauge field and a new massive gauge field, the authors of Ref. [11] analyzed the impurity effect in a holographic superconductor and observed that the mass gap in the optical conductivity disappears when the coupling is sufficiently large. More recently, $\mathrm{Hu}$ et al. constructed the holographic Josephson junction from the massive gravity and found that the increasing graviton mass parameter will change the superconductor phase into a normal metal phase for a fixed temperature (chemical potential), which indicates that the graviton mass parameter and the doping have a similar effect as from superconductivity to a normal metal [12]. Considering a holographic superconductor with a scalar field coupled kinematically to the Einstein tensor, Kuang and Papantonopoulos observed that, as the strength of the coupling increases, the critical temperature below which the scalar field condenses is lowering, the condensation gap decreases faster than the temperature, the width of the condensation gap is not proportional to the size of the condensate and at low temperatures the condensation gap tends to zero for the strong coupling, which suggests that the derivative coupling in the gravity bulk can have a dual interpretation on the boundary corresponding to impurities concentrations in a real material [13]. This model was further studied beyond the probe limit $[14,15]$. Other investigations based on the impurity effect on the holographic dual models can be found, for example, in Refs. [16-19].

On the other hand, since Veselago proposed in theory that the refractive index might be negative in some special material [20], there have been many investigations concerning this exotic electromagnetic phenomenon-negative refraction, which indicates that electromagnetic waves propagate in a direction opposite to that of the flow of energy in the so- 
called "metamaterials" [21-23]. Interestingly, it is believed that the superconductors are the ideal candidates for metamaterials due to their low loss, compact structure, extraordinary degree of nonlinearity and tunability, magnetic flux quantization and the Josephson effect, quantum effects in which photons interact with quantized energy levels in the meta-atom, and strong diamagnetism $[24,25]$. With the help of the AdS/CFT correspondence, the refractive index in the superconductors was investigated by holography in the probe limit [26] and away from the probe limit [27], which shows that in the superconducting phase there is negative refraction at low enough frequencies only in the backreacted case. It is interesting to note that Mahapatra et al. [28] constructed the generalized holographic superconductors via the Stückelberg mechanism [29-31] in the four dimensional R-charged black hole and observed that the superconducting phase can exhibit a negative Depine-Lakhtakia (DL) index [32] at low frequencies and below a cut-off value of the charge parameter even in the probe limit. Extending the investigation to the generalized superconductors with Born-Infeld electrodynamics, Cheng et al. found that the system has a negative DL index in the superconducting phase at small frequencies and the greater the Born-Infeld corrections the larger the range of frequencies or the range of temperatures for which the negative refraction occurs [33]. The refractive index in the holographic dual models can also be found, for example, in Refs. [34-44].

In this work, motivated by the recent studies in Refs. $[12,13]$, we will use the AdS/CFT correspondence to investigate systematically the effect of the doping or impurity on the superconductors in strongly coupled condensed matter systems and their negative refraction in the probe limit. In order to obtain the rich phase structure, we construct the generalized superconductors from the coupling of a scalar field to the Einstein tensor in the massive gravity via the Stuickelberg mechanism just as in [28]. We will observe that the larger graviton mass and Einstein tensor coupling parameters both hinder the formation of the condensation, but the larger graviton mass or smaller coupling parameter makes it easier for the emergence of the so-called Cave of Winds [45], i.e., the second-order transition occurs before the first-order transition to a new superconducting phase when the temperature decreases [46-48]. Furthermore, we assume that the boundary theory is weakly coupled to a dynamical electromagnetic field and one can calculate the refractive index of the system perturbatively. We will find that the larger graviton mass parameter or smaller coupling parameter makes the range of frequencies or the range of temperatures larger for which a negative DL index occurs, but the larger graviton mass and coupling parameters both can reduce the dissipation and improve the propagation in the holographic setup.

The organization of this work is as follows. In Sec. 2 we will use the AdS/CFT correspondence to construct the gen- eralized superconductors from the coupling of a scalar field to the Einstein tensor in the massive gravity. In Sec. 3 we will discuss the effects of the graviton mass and Einstein tensor coupling parameters on the scalar condensation and phase transition in the generalized holographic models. In Sec. 4 we will analyze the effects of the graviton mass and Einstein tensor coupling parameters on the negative refraction in the generalized holographic models. We will conclude in the last section of our main results.

\section{Generalized superconductors from the coupling of a scalar field to the Einstein tensor in massive gravity}

Since we are interested in the background of a massive gravity theory, we start with the following action for a fourdimensional ghost-free dRGT massive gravity $[49,50]$ :

$$
S=\frac{1}{16 \pi G} \int d^{4} x \sqrt{-g}\left[R+\frac{6}{L^{2}}+m^{2} \sum_{i}^{4} c_{i} \mathcal{U}_{i}(g, \mathrm{f})\right],
$$

where $\mathrm{f}$ is the reference metric, $m$ is the graviton mass parameter, $c_{i}$ are constants and $\mathcal{U}_{i}$ are symmetric polynomials of the eigenvalues of the matrix $\mathcal{K}_{v}^{\mu} \equiv \sqrt{g^{\mu \alpha} \mathrm{f}_{\alpha \nu}}$

$$
\begin{aligned}
& \mathcal{U}_{1}=[\mathcal{K}] \\
& \mathcal{U}_{2}=[\mathcal{K}]^{2}-\left[\mathcal{K}^{2}\right], \\
& \mathcal{U}_{3}=[\mathcal{K}]^{3}-3[\mathcal{K}]\left[\mathcal{K}^{2}\right]+2\left[\mathcal{K}^{3}\right], \\
& \mathcal{U}_{4}=[\mathcal{K}]^{4}-6\left[\mathcal{K}^{2}\right][\mathcal{K}]^{2}+8\left[\mathcal{K}^{3}\right][\mathcal{K}]+3\left[\mathcal{K}^{2}\right]^{2}-6\left[\mathcal{K}^{4}\right],
\end{aligned}
$$

where the square root in $\mathcal{K}$ means $(\sqrt{A})_{\nu}^{\mu}(\sqrt{A})_{\lambda}^{\nu}=A_{\lambda}^{\mu}$ and $[\mathcal{K}]=K_{\mu}^{\mu}$. The equation of motion of this action will be

$R_{\mu \nu}-\frac{1}{2} R g_{\mu \nu}-\frac{3}{L^{2}} g_{\mu \nu}+m^{2} \chi_{\mu \nu}=8 \pi G T_{\mu \nu}$,

with

$$
\begin{aligned}
\chi_{\mu \nu}= & -\frac{c_{1}}{2}\left(\mathcal{U}_{1} g_{\mu \nu}-\mathcal{K}_{\mu \nu}\right)-\frac{c_{2}}{2}\left(\mathcal{U}_{2} g_{\mu \nu}\right. \\
& \left.-2 \mathcal{U}_{1} \mathcal{K}_{\mu \nu}+2 \mathcal{K}_{\mu \nu}^{2}\right)-\frac{c_{3}}{2}\left(\mathcal{U}_{3} g_{\mu \nu}-3 \mathcal{U}_{2} \mathcal{K}_{\mu \nu}\right. \\
& \left.+6 \mathcal{U}_{1} \mathcal{K}_{\mu \nu}^{2}-6 \mathcal{K}_{\mu \nu}^{3}\right) \\
& -\frac{c_{4}}{2}\left(\mathcal{U}_{4} g_{\mu \nu}-4 \mathcal{U}_{3} \mathcal{K}_{\mu \nu}+12 \mathcal{U}_{2} \mathcal{K}_{\mu \nu}^{2}\right. \\
& \left.-24 \mathcal{U}_{1} \mathcal{K}_{\mu \nu}^{3}+24 \mathcal{K}_{\mu \nu}^{4}\right) .
\end{aligned}
$$

We consider the following reference metric [49]

$\mathrm{f}_{\mu \nu}=\operatorname{diag}\left(0,0, c_{0}^{2} h_{i j}\right)$,

with $c_{0}$ being a positive constant, and a general black hole solution is given by [50]

$d s^{2}=-r^{2} f(r) d t^{2}+\frac{1}{r^{2} f(r)} d r^{2}+r^{2} h_{i j} d x^{i} d x^{j}$, 
with

$f(r)=\frac{\kappa}{r^{2}}+\frac{1}{L^{2}}-\frac{m_{0}}{r^{3}}+\frac{c_{1} m^{2}}{2 r}+\frac{c_{2} m^{2}}{r^{2}}$,

where $m_{0}$ is related to the mass of the black hole, and $h_{i j} d x^{i} d x^{j}$ is the line element for the two-dimensional spherical, flat or hyperbolic space with $\kappa=-1,0$ or 1, respectively. In this work we are only interested in the planesymmetric black hole, so we will set $\kappa=0$ and the corresponding Hawking temperature is determined by

$T_{H}=\frac{1}{4 \pi r_{h}}\left(\frac{3 r_{h}^{2}}{L^{2}}+c_{1} m^{2} r_{h}+c_{2} m^{2}\right)$,

where $r_{h}$ is the radius of the event horizon. For this considered solution, the nonzero components of the Einstein tensor $G^{\mu \nu}$ are

$$
\begin{aligned}
G^{t t} & =-\frac{1}{r^{2}}\left(3+\frac{r f^{\prime}}{f}\right), \\
G^{r r} & =r^{2} f^{2}\left(3+\frac{r f^{\prime}}{f}\right), \\
G^{x x} & =G^{y y}=\frac{1}{2 r^{2}}\left[6 f+r\left(6 f^{\prime}+r f^{\prime \prime}\right)\right],
\end{aligned}
$$

where the prime denotes a derivative with respect to $r$.

In order to construct the generalized superconductors from the coupling of a scalar field to the Einstein tensor in the probe limit, we begin with the action describing a Maxwell field and a charged complex scalar field coupled to the Einstein tensor $G^{\mu \nu}[13]$

$$
\begin{aligned}
S= & \int d^{4} x \sqrt{-g}\left\{-\frac{F_{\mu \nu} F^{\mu \nu}}{4}\right. \\
& -\frac{\left(g^{\mu \nu}+\eta G^{\mu \nu}\right)\left[\left(\partial_{\mu}-i q A_{\mu}\right) \psi\right]\left[\left(\partial_{\nu}-i q A_{\nu}\right) \psi\right]^{*}}{2} \\
& \left.-\frac{m_{\psi}^{2}|\psi|^{2}}{2}\right\} .
\end{aligned}
$$

Just as in Ref. [30], rewriting the charged complex scalar field $\psi=\Psi e^{i \alpha}$ with real modulus $\Psi$ and phase $\alpha$, we can obtain the generalized action via the Stückelberg mechanism:

$$
\begin{aligned}
S= & \int d^{4} x \sqrt{-g}\left[-\frac{F_{\mu \nu} F^{\mu \nu}}{4}-\frac{\left(g^{\mu \nu}+\eta G^{\mu \nu}\right)\left(\partial_{\mu} \Psi\right)\left(\partial_{\nu} \Psi\right)}{2}\right. \\
& -\frac{m_{\Psi}^{2} \Psi^{2}}{2} \\
& \left.-|\mathrm{G}(\Psi)|\left(g^{\mu \nu}+\eta G^{\mu \nu}\right)\left(\partial_{\mu} \alpha-q A_{\mu}\right)\left(\partial_{\nu} \alpha-q A_{\nu}\right)\right],
\end{aligned}
$$

where $q$ and $m_{\Psi}$ are the charge and mass of the scalar field, the local $U(1)$ gauge symmetry is given by $q A_{\mu} \rightarrow$ $q A_{\mu}+\partial_{\mu} \lambda$ together with $\alpha \rightarrow \alpha+\lambda$, and $\mathrm{G}(\Psi)$ is the function of $\Psi$ and we take its absolute value to avoid a wrong sign for the kinetic term. Multiplying this action by $q^{2}$ and rescaling the bulk fields $\Psi, A_{\mu}$ and model parameter $\xi$ as $\Psi / q, A_{\mu} / q$ and $q^{n-2} \xi$ for the general function $\mathrm{G}(\Psi)=\Psi^{2}+\xi \Psi^{n}$, we can recover the action describing the generalized holographic superconductors of the Einstein's general relativity (GR) investigated in Ref. [28] when the coupling parameter $\eta \rightarrow 0$ and the graviton mass $m \rightarrow 0$. Without loss of generality, we will scale the AdS radius $L=1$ and the charge $q=1$ in the following calculation, just as in Ref. [13].

Using the gauge symmetry to fix the phase $\alpha=0$, from the action (11) we can obtain equations of motion for the matter fields:

$$
\begin{aligned}
& \frac{1}{\sqrt{-g}} \partial_{\mu}\left[\sqrt{-g}\left(g^{\mu \nu}+\eta G^{\mu \nu}\right)\left(\partial_{\nu} \Psi\right)\right]-m_{\Psi}^{2} \Psi \\
& -\frac{d \mathrm{G}(\Psi)}{d \Psi}\left(g^{\mu \nu}+\eta G^{\mu \nu}\right) A_{\mu} A_{v}=0, \\
& \frac{1}{\sqrt{-g}} \partial_{\mu}\left(\sqrt{-g} F^{\mu \nu}\right)-2 \mathrm{G}(\Psi)\left(g^{\mu \nu}+\eta G^{\mu \nu}\right) A_{\mu}=0 .
\end{aligned}
$$

Taking the ansatz of the matter fields as $\Psi=\Psi(r)$ and $A=\Phi(r) d t$, under the solution (6), the above equations of motion can be rewritten into

$$
\begin{aligned}
& {\left[1+\eta\left(3 f+r f^{\prime}\right)\right] \Psi^{\prime \prime}+\left[\frac{4}{r}+\frac{f^{\prime}}{f}+\eta\left(r f^{\prime \prime}+11 f^{\prime}\right.\right.} \\
& \left.\left.\quad+\frac{r f^{\prime 2}}{f}+\frac{12 f}{r}\right)\right] \Psi^{\prime} \\
& \quad+\frac{1}{r^{2} f}\left\{-m_{\Psi}^{2} \Psi+\Phi^{2}\left[\frac{1}{r^{2} f}+\eta\left(\frac{3}{r^{2}}+\frac{f^{\prime}}{r f}\right)\right] \frac{d \mathrm{G}(\Psi)}{d \Psi}\right\} \\
& =0, \\
& \Phi^{\prime \prime}+\frac{2}{r} \Phi^{\prime}-\frac{2 \mathrm{G}(\Psi)}{r^{2} f}\left[1+\eta\left(3 f+r f^{\prime}\right)\right] \Phi=0 .
\end{aligned}
$$

Using the shooting method [51], we can solve Eqs. (14) and (15) numerically by doing integration from the horizon out to the infinity with the appropriate boundary conditions for $\Psi$ and $\Phi$. At the event horizon $r=r_{h}$, the regularity leads to the boundary conditions

$\Phi\left(r_{h}\right)=0, \quad \Psi^{\prime}\left(r_{h}\right)=\frac{m_{\Psi}^{2}}{r_{h}^{2} f^{\prime}\left(r_{h}\right)\left[1+\eta r_{h} f^{\prime}\left(r_{h}\right)\right]} \Psi\left(r_{h}\right)$.

At the infinity $r \rightarrow \infty$, the solutions behave like

$\Phi=\mu-\frac{\rho}{r}, \quad \Psi=\frac{\Psi_{-}}{r^{\Delta_{-}}}+\frac{\Psi_{+}}{r^{\Delta_{+}}}$,

with the characteristic exponent

$\Delta_{ \pm}=\frac{1}{2}\left(3 \pm \sqrt{9+\frac{4 m_{\Psi}^{2}}{1+3 \eta}}\right)$,

where $\mu$ and $\rho$ are interpreted as the chemical potential and charge density in the dual field theory, respectively. According to the AdS/CFT correspondence, provided $\Delta_{-}$is larger than the unitarity bound, both $\Psi_{-}$and $\Psi_{+}$can be normalizable and they can be used to define operators in the dual field 
theory $\Psi_{-}=\left\langle O_{-}\right\rangle$and $\Psi_{+}=\left\langle O_{+}\right\rangle$, respectively. Considering that we focus on the condensate for the operator $\left\langle O_{+}\right\rangle$, we will impose boundary condition $\Psi_{-}=0$ in this work.

\section{Scalar condensation and phase transition}

In this section, we will solve the system of coupled differential equations (14) and (15) numerically and then discuss the scalar condensation and phase transition of these generalized superconductors. For concreteness, we will set $m_{\Psi}^{2}=-2$ since the choices of the scalar field mass will not qualitatively modify our results. In order to introduce a general class of gravity duals to superconducting theories that exhibit both first- and second-order phase transitions at finite temperature in strongly interacting systems, we consider a particular form of $\mathrm{G}(\Psi)$ introduced in Refs. [28,33], i.e.,

$\mathrm{G}(\Psi)=\Psi^{2}+\xi \Psi^{8}$,

where the model parameter $\xi$ will provide richer physics in the phase transition. We also concentrate on the cases for the specific values of the parameter $\xi$ in our discussion, i.e., $\xi=0.2$ and $\xi=0.5$ in order to compare with the results given in Refs. [28,33]. As a matter of fact, the other choices will not qualitatively modify our results. For the convenience of numerics, we will set $c_{1}=r_{h}$ and $c_{2}=-r_{h}^{2} / 2$, and choose the range of graviton mass parameters $0 \leq m \leq 1.2$, just as in [12]. It is interesting to note that, from the equations of motion (14) and (15), there exist the useful scaling symmetries and the transformation of the relevant quantities

$$
\begin{aligned}
r & \rightarrow \lambda r, \quad(t, x, y) \rightarrow \lambda^{-1}(t, x, y), \quad c_{1} \rightarrow \lambda c_{1}, \quad c_{2} \\
& \rightarrow \lambda^{2} c_{2}, \\
\Psi & \rightarrow \Psi, \quad \Phi \rightarrow \lambda \Phi, \quad(T, \mu) \rightarrow \lambda(T, \mu), \quad \rho \\
& \rightarrow \lambda^{2} \rho, \quad \Psi_{ \pm} \rightarrow \lambda^{\Delta_{ \pm}} \Psi_{ \pm},
\end{aligned}
$$

where $\lambda$ is a real positive number. We will use the scaling symmetries to set $r_{h}=1$ when performing numerical calculations.

On the other hand, we have to calculate the grand potential $\Omega=-\mathcal{T} \mathcal{S}_{o s}$ of the bound state in order to obtain the important information about the phase transition of the system, where $\mathcal{S}_{o s}$ is the Euclidean on-shell action. Working in the grand canonical ensemble, we have

$$
\begin{aligned}
\mathcal{S}_{o s}= & \int d t d x d y d r\left\{-\frac{1}{2} \partial_{\mu}\left(\sqrt{-g} F^{\mu \nu} A_{\nu}\right)\right. \\
& +\frac{1}{2} A_{\nu} \partial_{\mu}\left(\sqrt{-g} F^{\mu \nu}\right)-\frac{1}{2} \partial_{\mu}\left[\sqrt { - g } \Psi \left(g^{\mu \nu}\right.\right. \\
& \left.\left.+\eta G^{\mu \nu}\right)\left(\partial_{\nu} \Psi\right)\right]+\frac{1}{2} \Psi \partial_{\mu}\left[\sqrt{-g}\left(g^{\mu \nu}+\eta G^{\mu \nu}\right)\left(\partial_{\nu} \Psi\right)\right] \\
& \left.-\frac{1}{2} m_{\Psi}^{2} \Psi^{2}-\mathrm{G}(\Psi)\left(g^{\mu \nu}+\eta G^{\mu \nu}\right) A_{\mu} A_{\nu}\right\}
\end{aligned}
$$

$$
\begin{aligned}
= & \frac{V}{2 \mathcal{T}}\left\{\left.r^{2} \Phi \Phi^{\prime}\right|_{r \rightarrow \infty}-\left.r^{4} f\left[1+\eta\left(3 f+r f^{\prime}\right)\right] \Psi \Psi^{\prime}\right|_{r \rightarrow \infty}\right. \\
& \left.+\int_{r_{h}}^{\infty} d r \sqrt{-g}\left[\Psi \frac{d \mathrm{G}(\Psi)}{d \Psi}\left(g^{\mu \nu}+\eta G^{\mu \nu}\right) A_{\mu} A_{v}\right]\right\} \\
= & \frac{V}{2 \mathcal{T}}\left[\mu \rho-\int_{r_{h}}^{\infty} \Psi \frac{d \mathrm{G}(\Psi)}{d \Psi} \frac{1+\eta\left(3 f+r f^{\prime}\right)}{f} \Phi^{2} d r\right],
\end{aligned}
$$

where we have used the integration $\int d t d x d y=\frac{V}{\mathcal{T}}$. Thus, we can express the grand potential in the superconducting phase as:

$$
\begin{aligned}
& \frac{\Omega_{S}}{V}=-\frac{\mathcal{T} \mathcal{S}_{O S}}{V}=-\frac{1}{2} \mu \rho \\
& +\frac{1}{2} \int_{r_{h}}^{\infty} \Psi \frac{d \mathrm{G}(\Psi)}{d \Psi} \frac{1+\eta\left(3 f+r f^{\prime}\right)}{f} \Phi^{2} d r .
\end{aligned}
$$

In the normal phase, i.e., $\Psi=0$, we can easily get the grand potential

$\frac{\Omega_{N}}{V}=-\frac{1}{2} \mu \rho$.

3.1 Effect of the graviton mass on the scalar condensation and phase transition

In Fig. 1, we plot the condensate and corresponding grand potential as a function of the chemical potential with the fixed coefficient $\xi$ for different values of the graviton mass $m$ in the case of the coupling parameter $\eta=0.0$ by solving the equations of motion (14) and (15) numerically. Obviously, we can see clearly from left two panels that, for a fixed model parameter $\xi$, the critical chemical potential $\mu_{c}$ increases with the increase of the graviton mass parameter, i.e., the critical temperature $T_{c}$ decreases as $m$ increases, which indicates that the larger graviton mass parameters will hinder the formation of the condensation. This agrees well with the findings in the first holographic Josephson junction from the massive gravity introduced in Ref. [12], i.e., the graviton mass parameter and the doping have a similar effect as the phase transition from superconductivity to a normal metal.

What is more interesting is the rich phase structure of this system. In the case of $\xi=0$ or small $\xi$, for example $\xi=$ 0.2 , the transition is of the second order and the condensate approaches zero as $\left\langle O_{2}\right\rangle \sim\left(\mu-\mu_{c}\right)^{1 / 2}$ for all values of $m$ considered here, which is supported by the behavior of the grand potential just as shown in the top-right panel for $m=1.2$. Note that this conclusion still holds in the cases of $m \leq 1.0$ with $\xi=0.5$. However, the story is obviously different if we consider the case of $m=1.2$ in the bottom two panels of Fig. 1. We find that, as the chemical potential is increased, i.e., the temperature is lowered, the condensate $\left\langle O_{+}\right\rangle$becomes multivalued, and there is at first a second order phase transition from the normal to a superconducting phase and then a first order phase transition between two superconducting phases, which is the Cave of Winds [45] 

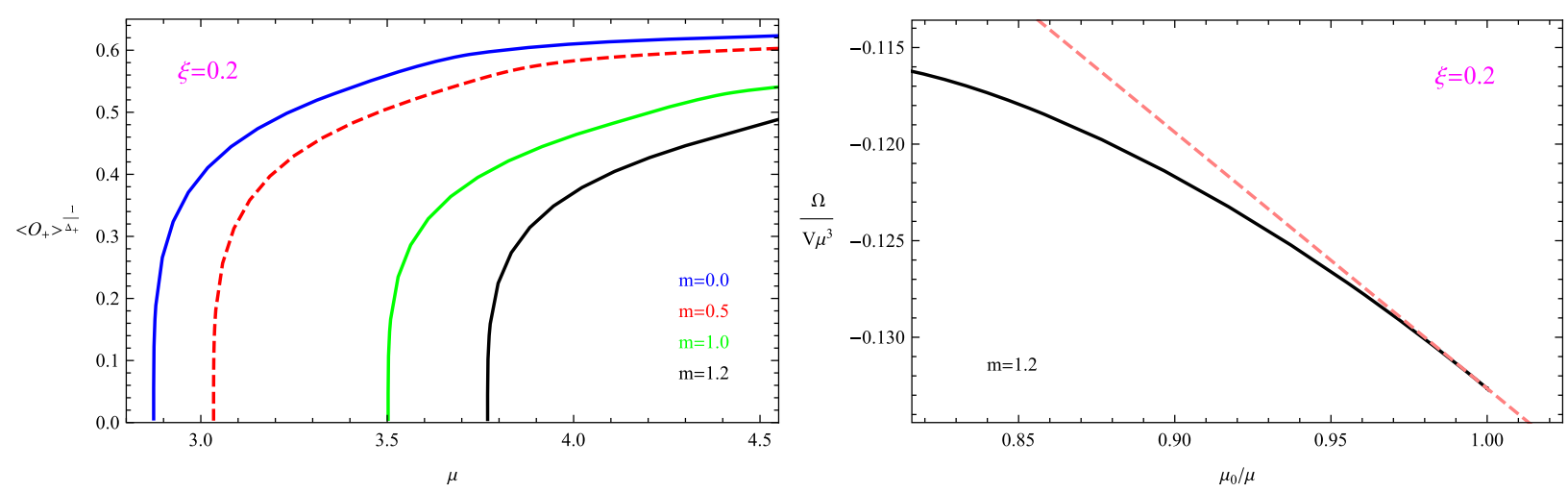

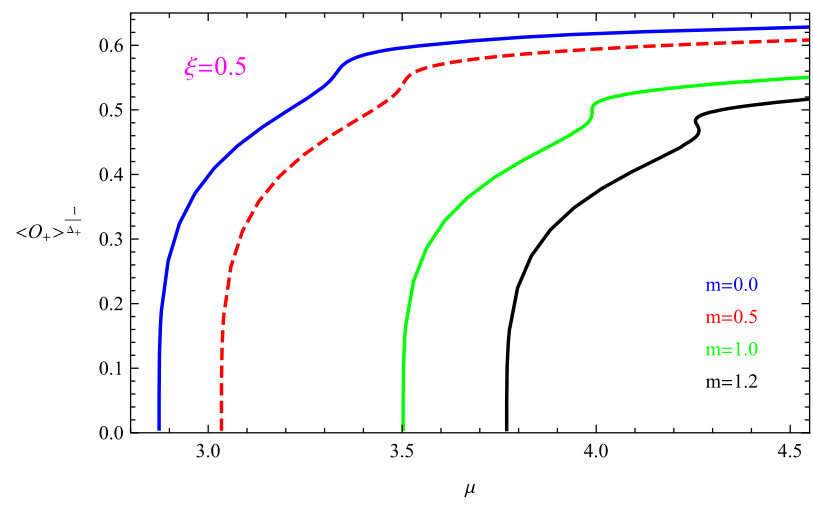

Fig. 1 The condensate and grand potential as a function of the chemical potential with the fixed coefficients $\xi=0.2$ (top) and $\xi=0.5$ (bottom) for different values of the graviton mass $m$ in the case of the coupling parameter $\eta=0.0$. For the left two panels, the four lines in each panel from left to right correspond to increasing graviton mass, i.e.,

and consistent with the behavior of the grand potential in the bottom-right panel. Thus, we conclude that the combination of the graviton mass and the model parameter provides richer physics in the phase transition, and the larger graviton mass makes it easier for the emergence of the Cave of Winds. It would be very interesting to see if there are any real systems with doping in the condensed matter which show this kind of Cave of Winds structure.

\subsection{Effect of the Einstein tensor on the scalar condensation and phase transition}

We now move on to the effect of the Einstein tensor on the scalar condensation and phase transition. Fixing the graviton mass by $m=1.0$, we exhibit the condensate and grand potential as a function of the chemical potential with the coefficient $\xi=0.2$ (top) and $\xi=0.5$ (bottom) for different values of the coupling parameter $\eta$ in Fig. 2. From left two panels, we observe that, similar to the effect of the graviton mass parameter, the critical chemical potential $\mu_{c}$ increases as the coupling parameter $\eta$ increases for a fixed model parameter $\xi$, which implies that the Einstein tensor will hinder the

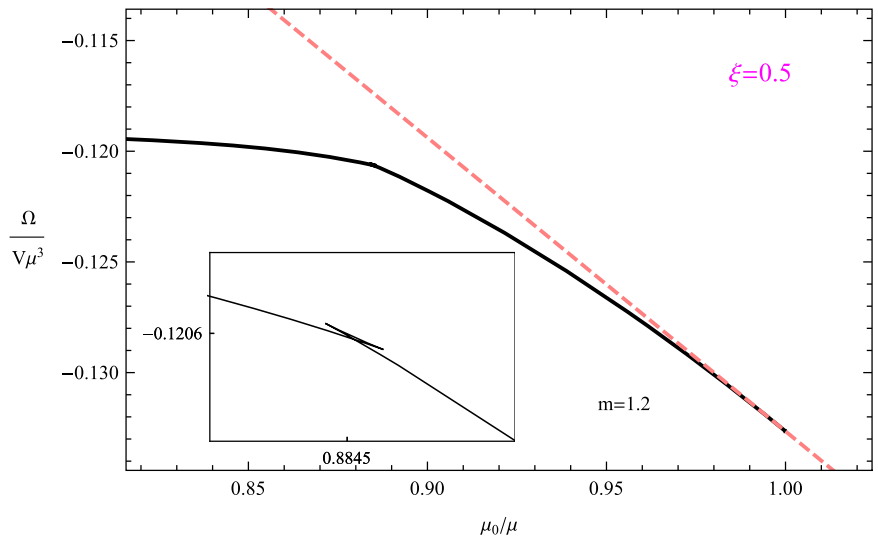

$m=0.0$ (blue), 0.5 (red dotted), 1.0 (green) and 1.2 (black), respectively. For the right two panels, the two lines in each panel correspond to the superconducting phase (black) and normal phase (pink dotted) in the case of $m=1.2$, respectively

condensate of the scalar field. This can be used to back up the observation obtained in Ref. [13] and suggests that this derivative coupling in the gravity bulk can have a dual interpretation on the boundary corresponding to impurities concentrations in a real material.

Focusing on the phase structure of this system, we find that the transition is of the second order and the condensate approaches zero as $\left\langle O_{2}\right\rangle \sim\left(\mu-\mu_{c}\right)^{1 / 2}$ for all values of $\eta$ considered here in the case of $\xi=0$ or small $\xi$, for example $\xi=0.2$, which is in good agreement with the behavior of the grand potential just as shown in the top-right panel for $\eta=-0.01$. Interestingly, in the case of large $\xi$, for example $\xi=0.5$, the conclusion still holds for the large coupling parameter, for example $\eta \geq 0.00$ considered here. But in the case of $\eta=-0.01$ with $\xi=0.5$, we show that, as the chemical potential is increased, the condensate $\left\langle O_{+}\right\rangle$becomes multivalued and the second-order transition occurs before the first-order transition to a new superconducting phase, which is supported by the behavior of the grand potential as shown in the bottom-right panel. Obviously, the smaller the coupling parameter the easier it is for the Cave of Winds to emerge, which is different from the effect of the graviton 

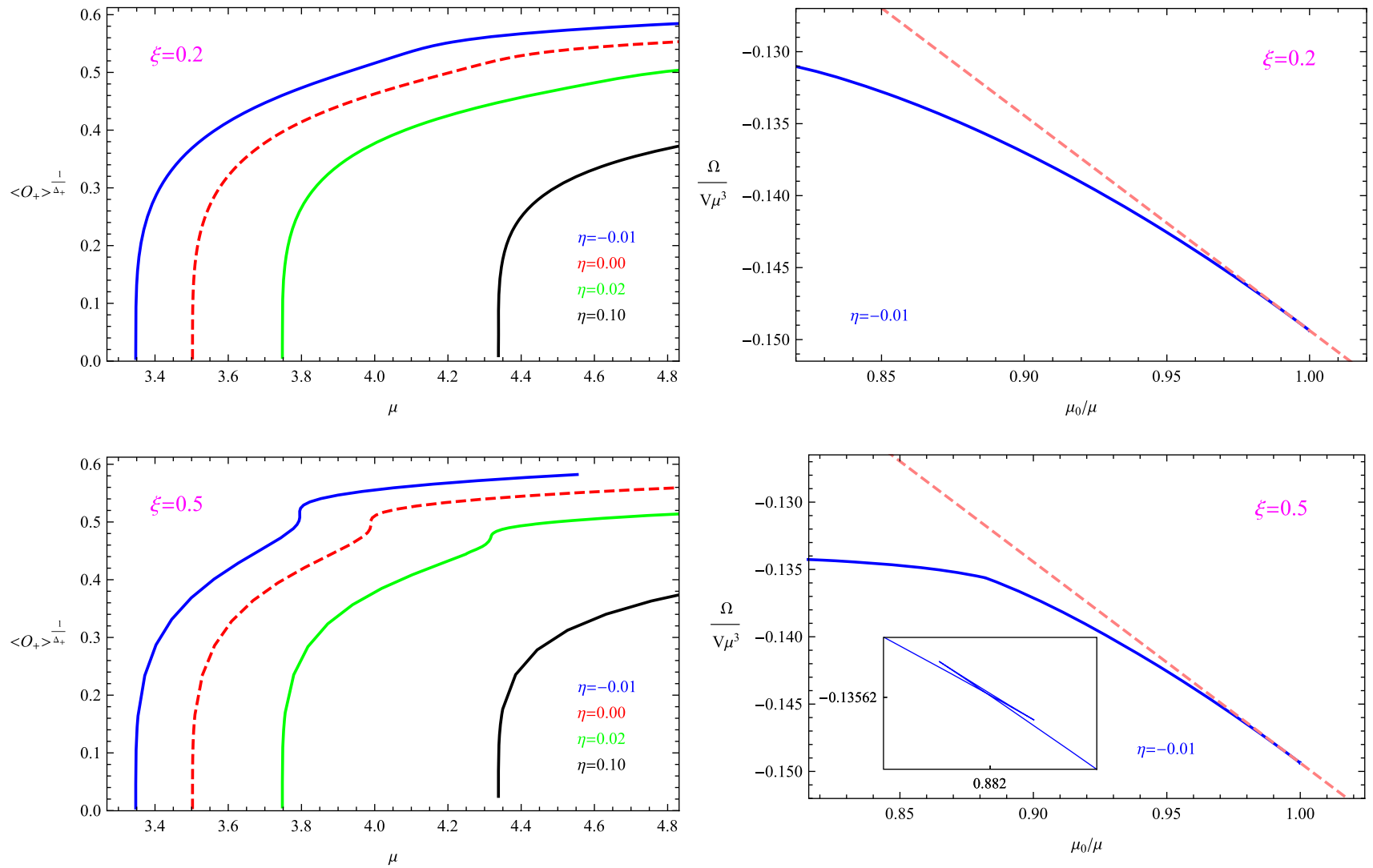

Fig. 2 The condensate and grand potential as a function of the chemical potential with the fixed coefficient $\xi=0.2$ (top) and $\xi=0.5$ (bottom) for different values of the coupling parameter $\eta$ in the case of the graviton mass $m=1.0$. For the left two panels, the four lines in each panel from top to bottom correspond to increasing coupling parameter,

mass parameter. Of course, it is worthwhile to see if there are any real systems with impurities in the condensed matter which present this kind of the Cave of Winds structure.

\section{Negative refractive index}

In previous section, we have revealed the effects of the graviton mass and Einstein tensor on the scalar condensation and phase transition, i.e., the graviton mass and Einstein tensor have the similar effect on the critical chemical potential but completely different effect on the emergence of the Cave of Winds. Now we are going to investigate the effects of the graviton mass and Einstein tensor on the optical properties of the generalized superconductors, especially on the negative refraction in these holographic systems.

Before proceeding, we first briefly review the electric permittivity $\epsilon(\omega)$, effective magnetic permeability $\mu(\omega)$ and their dependence on the retarded correlators in the linear response theory investigated by Amariti et al. [34]. Expanding the transverse part of the dielectric tensor for the small wave vector $k$ as i.e., $\eta=-0.01$ (blue), 0.00 (red dotted), 0.02 (green) and 0.10 (black), respectively. For the right two panels, the two lines in each panel correspond to the superconducting phase (blue) and normal phase (pink dotted) in the case of $\eta=-0.01$, respectively

$\epsilon_{T}(\omega, k)=\epsilon(\omega)+\frac{k^{2}}{\omega^{2}}\left[1-\frac{1}{\mu(\omega)}\right]+O\left(k^{4}\right)$,

one can obtain the refractive index for the isotropic media [34]

$n^{2}(\omega)=\frac{k^{2}}{\omega^{2}}=\epsilon(\omega) \mu(\omega)$.

With the form of $\epsilon_{T}(\omega, k)$ specified by the transverse part of the retarded current-current correlator $G_{T}(\omega, k)[52,53]$, i.e.,

$\epsilon_{T}(\omega, k)=1+\frac{4 \pi}{\omega^{2}} C_{e m}^{2} G_{T}(\omega, k)$,

and the expanded form of the retarded correlator in $k$ :

$G_{T}(\omega, k)=G_{T}^{0}(\omega)+k^{2} G_{T}^{2}(\omega)+O\left(k^{4}\right)$,

the electric permittivity and effective magnetic permeability can be expressed as:

$$
\epsilon(\omega)=1+\frac{4 \pi}{\omega^{2}} C_{e m}^{2} G_{T}^{0}(\omega),
$$




$$
\mu(\omega)=\frac{1}{1-4 \pi C_{e m}^{2} G_{T}^{2}(\omega)},
$$

where $C_{e m}$ is the electromagnetic coupling constant which will be set to unity when performing numerical calculations, and $G_{T}^{0}(\omega)$ and $G_{T}^{2}(\omega)$ are the expansion coefficients of the retarded correlators [54], respectively.

It should be noted that, when taking the dissipation of the system into account, the electric permittivity $\epsilon$, magnetic permeability $\mu$ and refractive index $n$ are generally complex functions of the frequency $\omega$. In Ref. [55], McCall et al. derived the condition for the negative refraction in the dissipative medium and pointed out that it is not necessary for real parts $\operatorname{Re}(\epsilon)<0$ and $\operatorname{Re}(\mu)<0$ simultaneously. Later, Depine and Lakhtakia found that, for the dissipative medium, the sign of the refractive index coincide with the sign of the DL index [32]:

$n_{D L}=\operatorname{Re}[\epsilon(\omega)]|\mu(\omega)|+\operatorname{Re}[\mu(\omega)]|\epsilon(\omega)|$,

where $n_{D L}<0$ indicates that the phase velocity in the medium is opposite to the direction of the energy flow, i.e., the system has a negative index of refraction. As a simple, convenient, and widely adopted criterion, we will use the DL index $n_{D L}$ to check if the medium has positive or negative refraction in this work.

In order to compute the refractive index of our holographic systems, we will use the AdS/CFT correspondence to derive the expression of $G_{T}^{0}(\omega)$ and $G_{T}^{2}(\omega)$. Assuming that the perturbed Maxwell field has a form $\delta A_{x}=A_{x}(r) e^{-i \omega t+i k y}$, we get the equation of motion:

$$
\begin{aligned}
& A_{x}^{\prime \prime}+\left(\frac{2}{r}+\frac{f^{\prime}}{f}\right) A_{x}^{\prime}+\frac{1}{r^{2} f}\left\{\frac{\omega^{2}}{r^{2} f}-\frac{k^{2}}{r^{2}}\right. \\
& \left.-\mathrm{G}(\Psi)\left[2+\eta\left(6 f+6 r f^{\prime}+r^{2} f^{\prime \prime}\right)\right]\right\} A_{x}=0 .
\end{aligned}
$$

Taking the same series expansion of $A_{x}$ as in Eq. (27) for $G_{T}(\omega, k)$, i.e.,

$$
A_{x}(r)=A_{x 0}(r)+k^{2} A_{x 2}(r)+O\left(k^{4}\right),
$$

from Eq. (30) we arrive at the equations of motion for $A_{x 0}$ and $A_{x 2}$

$$
\begin{aligned}
& A_{x 0}^{\prime \prime}+\left(\frac{2}{r}+\frac{f^{\prime}}{f}\right) A_{x 0}^{\prime}+\frac{1}{r^{2} f}\left\{\frac{\omega^{2}}{r^{2} f}-\mathrm{G}(\Psi)[2+\eta(6 f\right. \\
& \left.\left.\left.+6 r f^{\prime}+r^{2} f^{\prime \prime}\right)\right]\right\} A_{x 0}=0, \\
& A_{x 2}^{\prime \prime}+\left(\frac{2}{r}+\frac{f^{\prime}}{f}\right) A_{x 2}^{\prime}+\frac{1}{r^{2} f}\left\{\frac{\omega^{2}}{r^{2} f}-\mathrm{G}(\Psi)[2+\eta(6 f\right. \\
& \left.\left.\left.+6 r f^{\prime}+r^{2} f^{\prime \prime}\right)\right]\right\} A_{x 2}-\frac{A_{x 0}}{r^{4} f}=0,
\end{aligned}
$$

which can be numerically solved by the ingoing wave boundary conditions near the horizon:

$A_{x 0} \propto f^{-\frac{i \omega}{\left(3+m^{2} / 2\right) r_{h}}}, \quad A_{x 2} \propto f^{-\frac{i \omega}{\left(3+m^{2} / 2\right) r_{h}}}$,

and the behaviors near the asymptotic AdS boundary:

$A_{x 0}=A_{x 0}^{(0)}+\frac{A_{x 0}^{(1)}}{r}, \quad A_{x 2}=A_{x 2}^{(0)}+\frac{A_{x 2}^{(1)}}{r}$.

Thus, according to the AdS/CFT dictionary, we obtain the retarded correlators:

$G_{T}^{0}(\omega)=\frac{A_{x 0}^{(1)}}{A_{x 0}^{(0)}}, \quad G_{T}^{2}(\omega)=\frac{A_{x 0}^{(1)}}{A_{x 0}^{(0)}}\left[\frac{A_{x 2}^{(1)}}{A_{x 0}^{(1)}}-\frac{A_{x 2}^{(0)}}{A_{x 0}^{(0)}}\right]$,

which can be used to calculate $\epsilon(\omega)$ and $\mu(\omega)$ in Eq. (28) and $n_{D L}$ in Eq. (29). 4.1 Effect of the graviton mass on the negative refractive
index

Let us firstly consider the effect of the graviton mass $m$ on the negative refraction. Setting the coupling parameter $\eta=0$, in Figs. 3 and 4 we plot the real and imaginary parts of the permittivity $\epsilon$ and permeability $\mu$ as a function of $\omega / T$ for different values of the graviton mass parameter $m$. Since the system has negative refractive index in the range $0.81 T_{c} \leq$ $T \leq 0.85 T_{c}$, we will fix the temperatures by $T=0.81 T_{c}$ and $T=0.85 T_{c}$ in the numerical computation. From the figures, we find that, for the fixed temperatures $T$ and all values of $m$ considered here, $\operatorname{Re}(\epsilon)$ and $\operatorname{Im}(\mu)$ are always negative but $\operatorname{Im}(\epsilon)$ and $\operatorname{Re}(\mu)$ are positive at low frequencies. Just as pointed out in Ref. [34], the refraction may be negative when $\operatorname{Re}(\epsilon)$ and $\operatorname{Re}(\mu)$ are not simultaneously negative because of the presence of the imaginary parts. It should be noted that $\operatorname{Im}(\mu)<0$ could imply some problem in the $\epsilon-\mu$ approach $[27,28,40]$, although Markel argued that $\operatorname{Im}(\mu)$ can in fact be negative for diamagnetic materials [56]. We will leave this point for further study since this delicate issue goes beyond the scope of our paper.

In order to obtain the effect of the graviton mass $m$ on the negative refraction, we plot the behavior of the DL index $n_{D L}$ as a function of $\omega / T$ with the fixed temperatures $T=0.81 T_{c}$ and $T=0.85 T_{c}$ for different values of $m$ in Fig. 5. Obviously, we observe the existence of the negative DL index below a certain value of $\omega / T$ with the fixed temperatures $T$ for all values of $m$ considered here. For the fixed temperature, we see that the critical value of $\omega / T$, below which the negative $n_{D L}$ appears, increases with the increase of the graviton mass $m$, which implies that the larger graviton mass makes the range of frequencies larger for which negative refraction is allowed. On the other hand, we notice that the emergence of the negative DL index becomes more obvious for the larger graviton mass in both cases, i.e., $T=0.81 T_{c}$ 

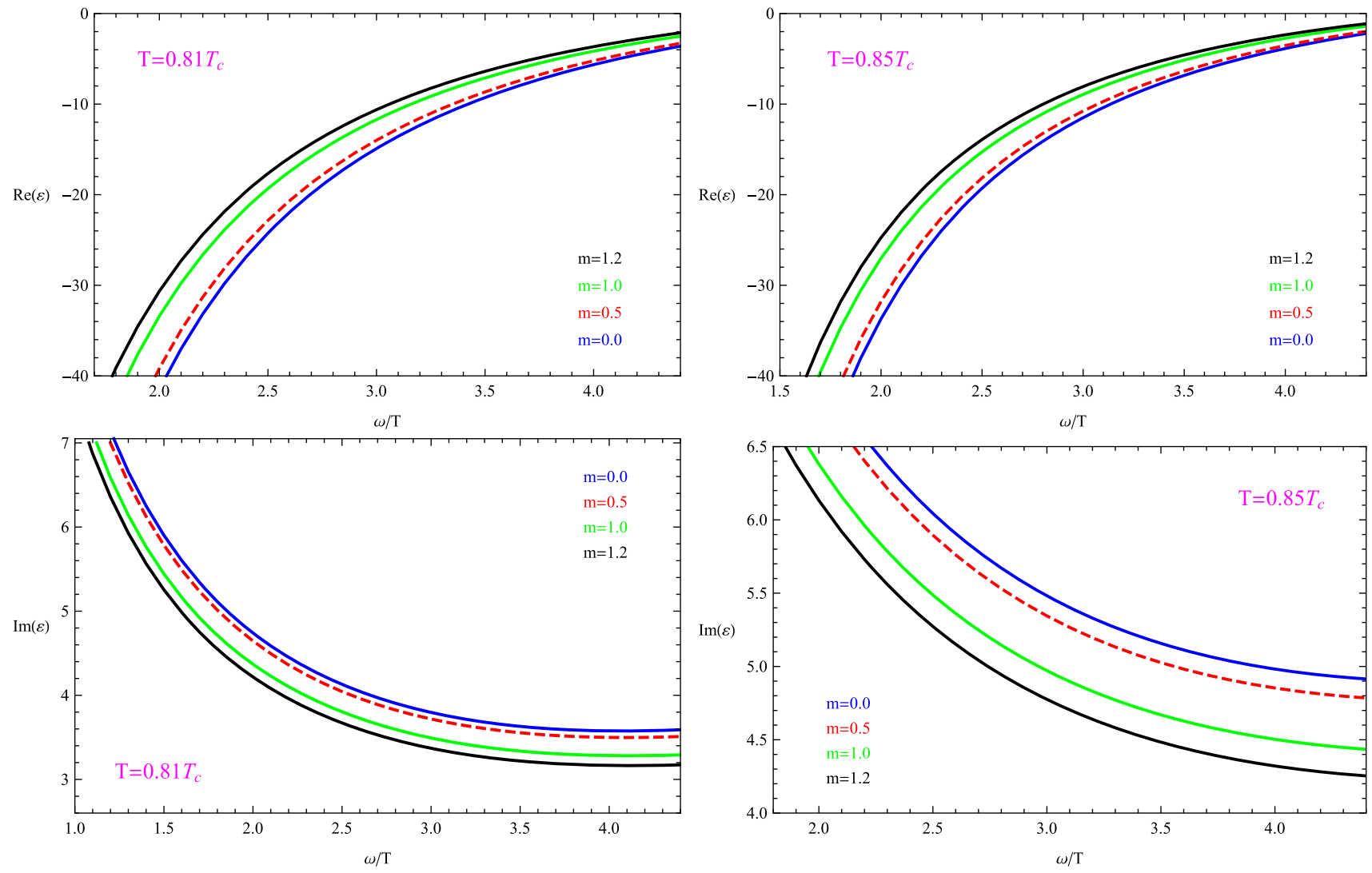

Fig. 3 Real (top) and imaginary (bottom) parts of the permittivity $\epsilon$ as a function of $\omega / T$ with the fixed temperatures $T=0.81 T_{c}$ (left) and $T=0.85 T_{c}$ (right) for different values of the graviton mass parame-

ter, i.e., $m=0.0$ (blue), 0.5 (red dotted), 1.0 (green) and 1.2 (black), respectively. We have set the coupling parameter $\eta=0$ in the numerical computation

and $T=0.85 T_{c}$, which indicates that the system with the larger graviton mass has a negative DL index in the superconducting phase even $T<0.81 T_{c}$ or $T>0.85 T_{c}$. This means that the larger graviton mass also makes the range of temperatures larger for which negative refraction appears. Thus, it is interesting to note that we can use the graviton mass $m$ to accommodate the range of frequencies and the range of temperatures for which negative refraction occurs.

Note that $\left|k^{2} G_{T}^{2}(\omega) / G_{T}^{0}(\omega)\right| \ll 1$ is the validity condition of Eq. (27). With the help of Eq. (25), we present in Fig. 6 the curves of $\left|\frac{G_{T}^{2}(\omega)}{G_{T}^{0}(\omega)} n^{2}\right| \omega^{2}$ as a function of $\omega / T$ with the fixed temperatures $T=0.81 T_{c}$ and $T=0.85 T_{c}$ for different values of the graviton mass parameter $m$. Due to the appearance of a negative imaginary part of the magnetic permeability, the validity condition is not very strictly satisfied, just as shown in Fig. 6, which may be an unfortunate feature in the probe limit $[28,40]$. However, we find that within the negative refraction frequency range $\left|\frac{G_{T}^{2}(\omega)}{G_{T}^{0}(\omega)} n^{2}\right| \omega^{2}$ is always less than one for the fixed temperatures $T$ and all values of $m$ considered here, which means that the validity condition gets marginally satisfied. Thus, our results are reliable.
It is also important to calculate the ratio $\operatorname{Re}(n) / \operatorname{Im}(n)$ and analyze the propagation $\operatorname{Re}(n)$ and the dissipation $\operatorname{Im}(n)$ of electromagnetic waves in systems. In Fig. 7, we plot the ratio $\operatorname{Re}(n) / \operatorname{Im}(n)$ as a function of $\omega / T$ with the fixed temperatures $T=0.81 T_{c}$ and $T=0.85 T_{c}$ for different values of the graviton mass parameter $m$. In both cases, i.e., $T=0.81 T_{c}$ and $T=0.85 T_{c}$, we observe that the magnitude of $\operatorname{Re}(n) / \operatorname{Im}(n)$ is small in the frequency range where $n_{D L}$ is negative, which indicates large dissipation in the system. However, for the fixed temperature, the ratio increases with increasing values of $m$ for the fixed $\omega / T$, which means that the larger graviton mass does enhance the magnitude of $\operatorname{Re}(n) / \operatorname{Im}(n)$ and thereby increases the propagation. Hence we can use the graviton mass to reduce the dissipation and improve the propagation in the holographic setup.

\subsection{Effect of the Einstein tensor on the negative refractive index}

Moving on to the effect of the Einstein tensor on the negative refraction, we firstly give the behaviors of the permittivity $\epsilon$ and permeability $\mu$ as a function of $\omega / T$ with the fixed tem- 

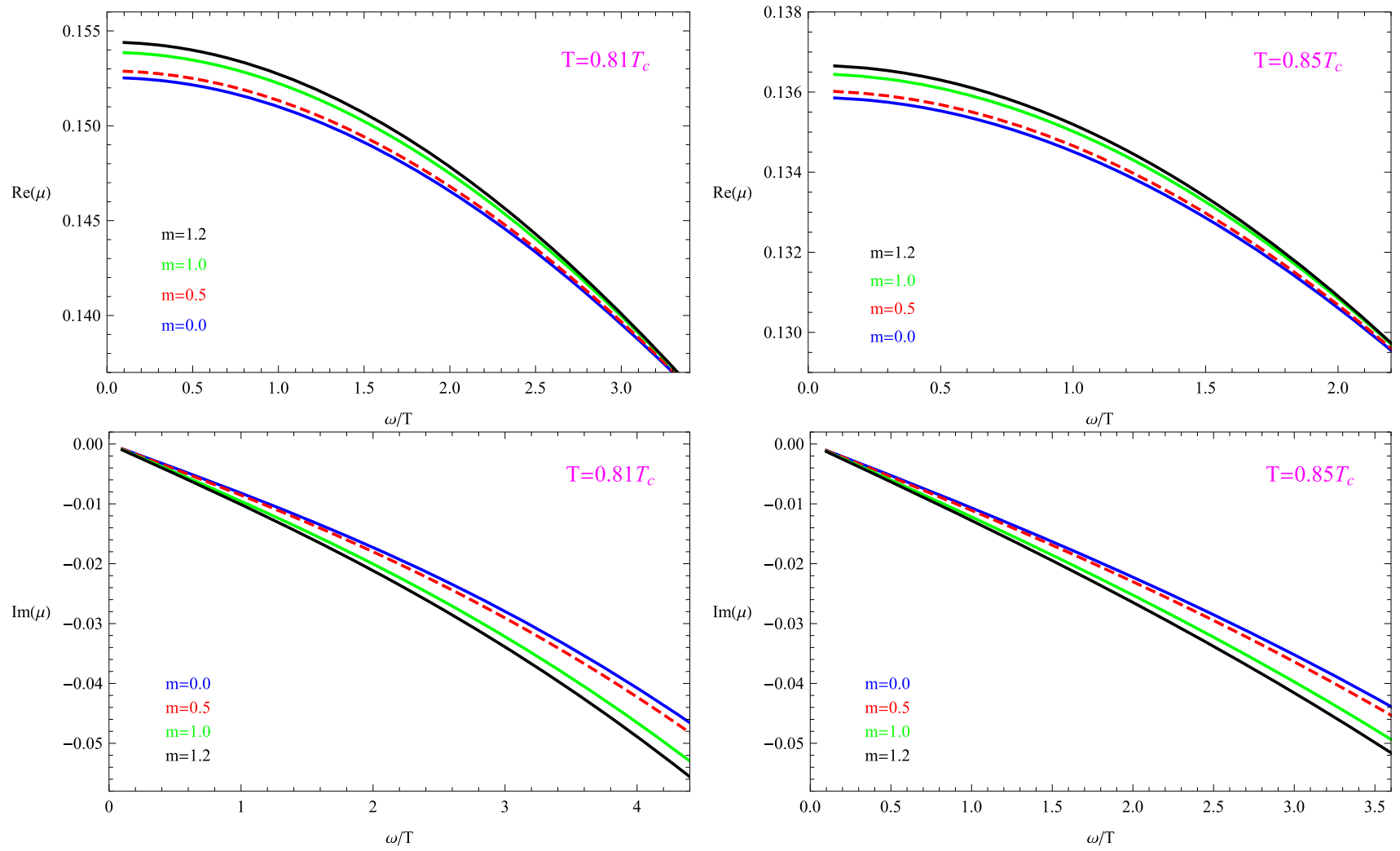

Fig. 4 Real (top) and imaginary (bottom) parts of the permeability $\mu$ as a function of $\omega / T$ with the fixed temperatures $T=0.81 T_{c}$ (left) and $T=0.85 T_{c}$ (right) for different values of the graviton mass parame-

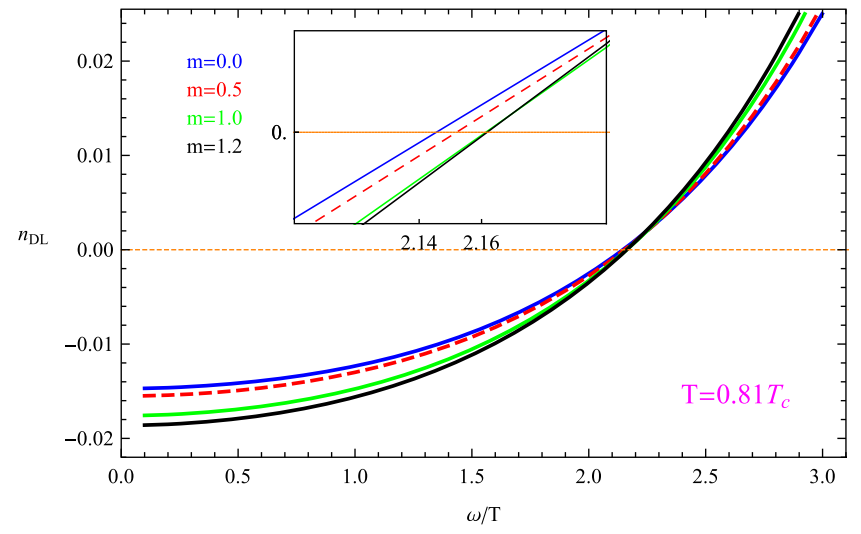

Fig. 5 Depine-Lakhtakia index $n_{D L}$ as a function of $\omega / T$ with the fixed temperatures $T=0.81 T_{c}$ (left) and $T=0.85 T_{c}$ (right) for different values of the graviton mass parameter, i.e., $m=0.0$ (blue), 0.5

peratures $T=0.81 T_{c}$ and $T=0.85 T_{c}$ for different values of the Einstein tensor coupling parameter in case of the graviton mass $m=1.0$ in Figs. 8 and 9. It is shown that, for the permittivity, $\operatorname{Re}(\epsilon)$ is negative at low frequencies and $\operatorname{Im}(\epsilon)$ is always positive, but for the permeability, $\operatorname{Re}(\mu)$ is always positive and $\operatorname{Im}(\mu)$ is negative, which implies that the refrac- ter, i.e., $m=0.0$ (blue), 0.5 (red dotted), 1.0 (green) and 1.2 (black), respectively. We have set the coupling parameter $\eta=0$ in the numerical computation

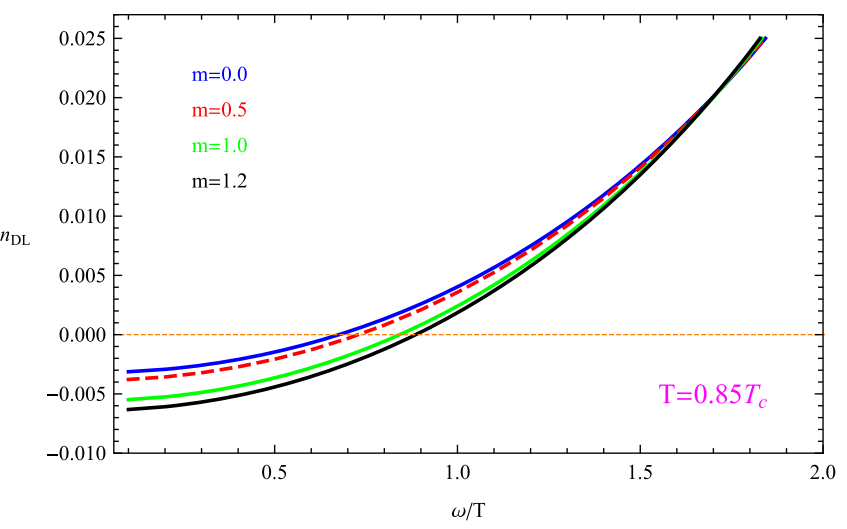

(red dotted), 1.0 (green) and 1.2 (black), respectively. We have set the coupling parameter $\eta=0$ in the numerical computation

tion may be negative since the real parts of the permittivity $\operatorname{Re}(\epsilon)$ and of the permeability $\operatorname{Re}(\mu)$ are not simultaneously negative. It should be noted that we have defined an effective magnetic permeability that is not an observable, although there is a caveat, i.e., the appearance of a negative imaginary 

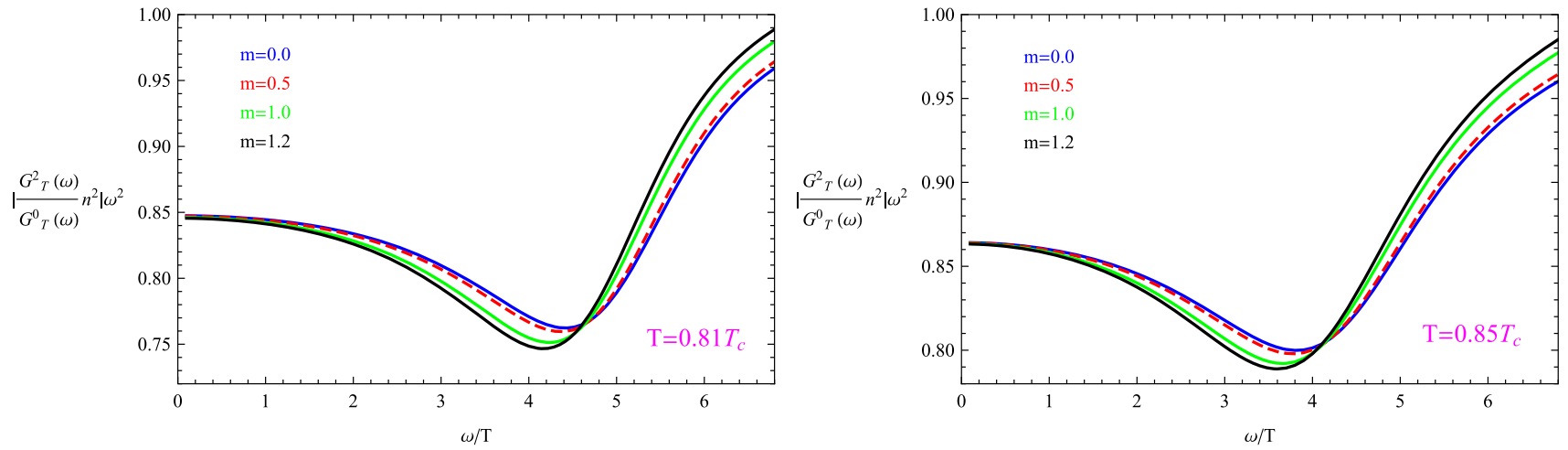

Fig. $6\left|\frac{G_{T}^{2}(\omega)}{G_{T}^{0}(\omega)} n^{2}\right| \omega^{2}$ as a function of $\omega / T$ with the fixed temperatures $T=0.81 T_{c}$ (left) and $T=0.85 T_{c}$ (right) for different values of the graviton mass parameter, i.e., $m=0.0$ (blue), 0.5 (red dotted), 1.0 (green) and 1.2 (black), respectively. We have set the coupling parameter $\eta=0$ in the numerical computation

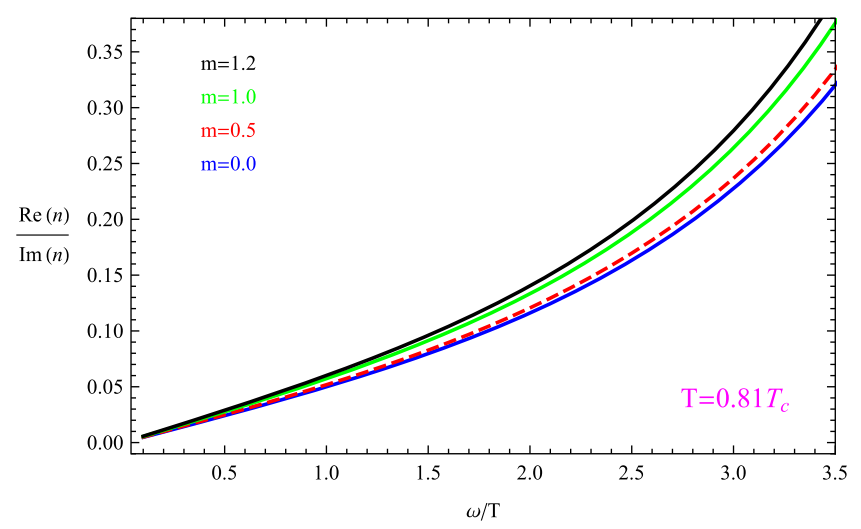

Fig. 7 Ratio $\operatorname{Re}(n) / \operatorname{Im}(n)$ as a function of $\omega / T$ with the fixed temperatures $T=0.81 T_{c}$ (left) and $T=0.85 T_{c}$ (right) for different values of the graviton mass parameter, i.e., $m=0.0$ (blue), 0.5 (red dotted), 1.0

part of the magnetic permeability. We will proceed with this point in mind.

In Fig. 10, we present the curves of the DL index $n_{D L}$ as a function of $\omega / T$ with the fixed temperatures $T=0.81 T_{c}$ and $T=0.85 T_{C}$ for different values of the coupling parameter $\eta$ in case of the graviton mass $m=1.0$. For the fixed temperature, we observe that the critical value of $\omega / T$, below which the system has negative $n_{D L}$, decreases with the increase of the coupling parameter $\eta$, which indicates that the larger Einstein tensor coupling parameter reduces the range of frequencies for which negative refraction occurs. On the other hand, we see that the negative $n_{D L}$ appears in the case of the coupling parameter $\eta=1.00$ at the temperature $T=0.81 T_{C}$ and disappears at $T=0.85 T_{c}$, but the negative $n_{D L}$ always appears in the case of the coupling parameter $\eta=0.10$ ( $\eta=0.00$ or $\eta=-0.01)$ at the temperatures $T=0.81 T_{c}$ and $T=0.85 T_{c}$, which means that the larger Einstein tensor coupling parameter also reduces the range of temperatures

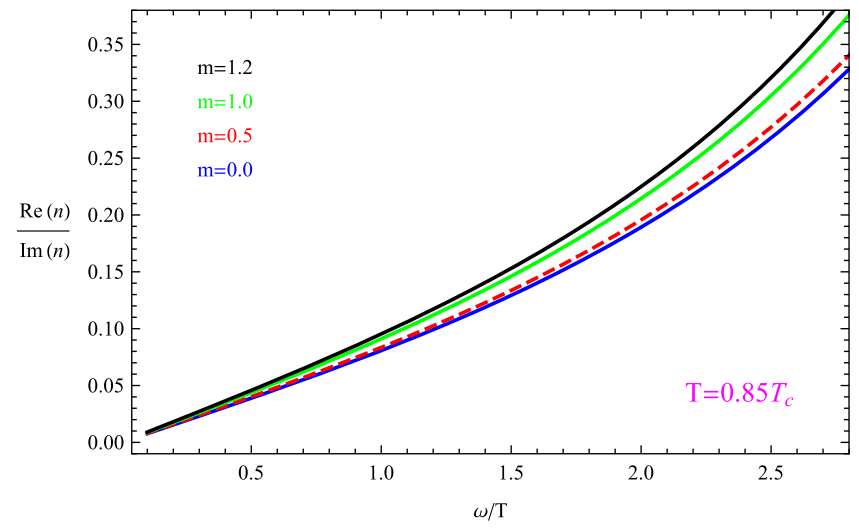

(green) and 1.2 (black), respectively. We have set the coupling parameter $\eta=0$ in the numerical computation

for which negative refraction occurs. The behavior of the Einstein tensor is reminiscent of that seen for the graviton mass, so we conclude that the graviton mass and Einstein tensor have completely different effects on the negative refractive index of our holographic systems.

In order to verify the validity of the expansion used in Eq. (27), we plot in Fig. 11 the behavior of $\left|\frac{G_{T}^{2}(\omega)}{G_{T}^{0}(\omega)} n^{2}\right| \omega^{2}$ as a function of $\omega / T$ with the fixed temperatures $T=0.81 T_{c}$ and $T=0.85 T_{c}$ for different values of the coupling parameter $\eta$ in case of the graviton mass $m=1.0$. It is shown that, within the negative refraction frequency range, the constraint $\left|k^{2} G_{T}^{2}(\omega) / G_{T}^{0}(\omega)\right| \ll 1$ is marginally satisfied for all values of $\eta$ considered here since the $\epsilon-\mu$ analysis is valid only for the frequencies for which this constraint is not violated.

Finally, we present in Fig. 12 the ratio $\operatorname{Re}(n) / \operatorname{Im}(n)$ as a function of $\omega / T$ with the fixed temperatures $T=0.81 T_{c}$ and $T=0.85 T_{C}$ for different values of the coupling parameter $\eta$ in case of the graviton mass $m=1.0$. Obviously, 

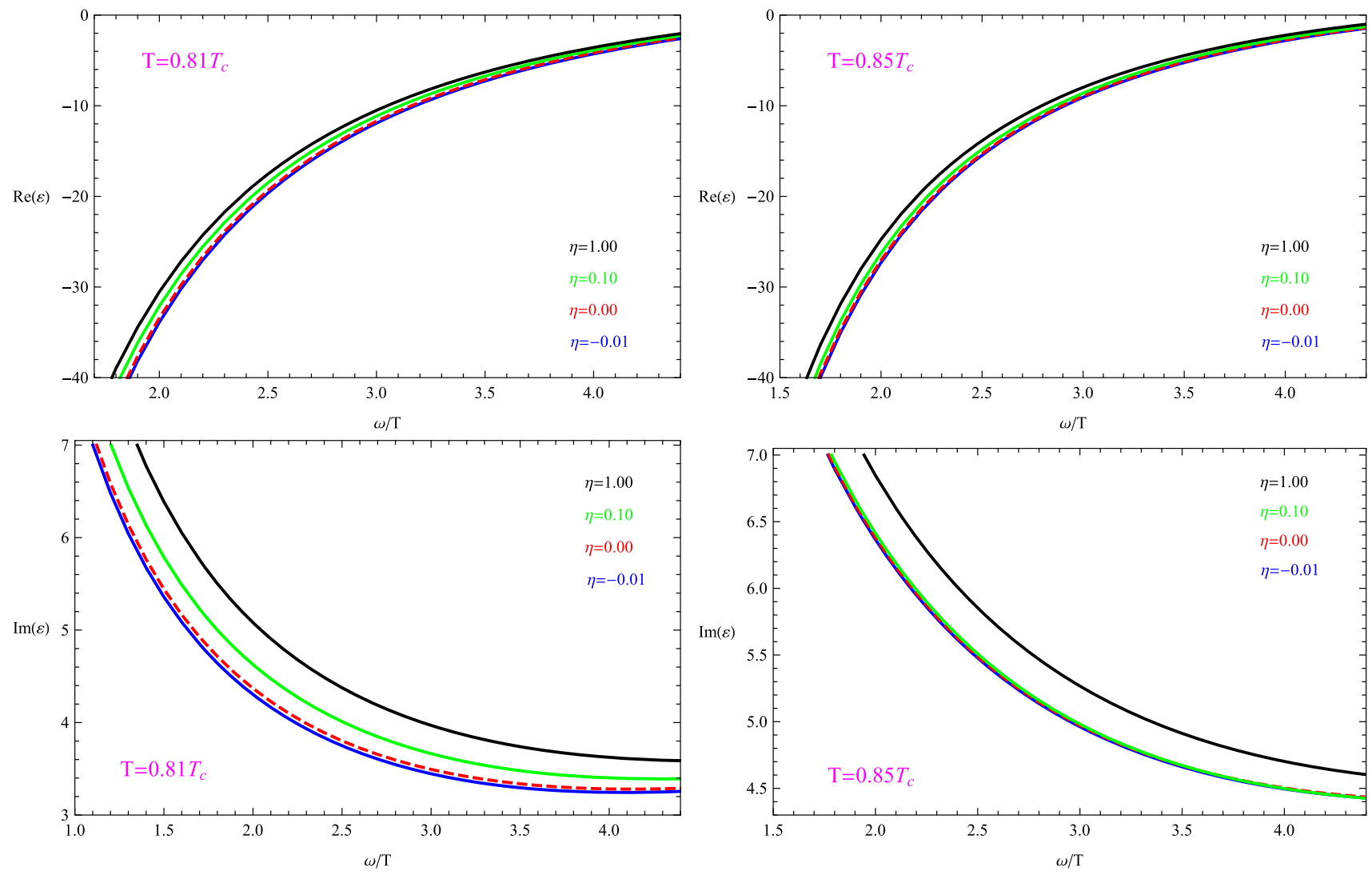

Fig. 8 Real (top) and imaginary (bottom) parts of the permittivity $\epsilon$ as a function of $\omega / T$ with the fixed temperatures $T=0.81 T_{c}$ (left) and $T=0.85 T_{c}$ (right) for different values of the coupling parameter,

i.e., $\eta=-0.01$ (blue), 0.00 (red dotted), 0.10 (green) and 1.00 (black), respectively. We have set the graviton mass $m=1.0$ in the numerical computation

for the fixed temperature, i.e., $T=0.81 T_{c}$ or $T=0.85 T_{c}$, the ratio increases with increasing values of $\eta$ for the fixed $\omega / T$, which indicates that the larger Einstein tensor coupling parameter enhances the magnitude of $\operatorname{Re}(n) / \operatorname{Im}(n)$ and can be used to improve the propagation in the holographic setup. Interestingly, the effect of the Einstein tensor on the ratio $\operatorname{Re}(n) / \operatorname{Im}(n)$ is similar to that of the graviton mass.

\section{Conclusions}

Recent studies showed that the graviton mass parameter has the similar effect on the phase transition from superconductivity to a normal metal as that of the doping [12] and the coupling of a scalar field to the Einstein tensor in the gravity bulk can have a dual interpretation on the boundary corresponding to impurities concentrations in a real material [13]. In order to investigate systematically the influence of the doping or impurity on the superconductors in strongly coupled condensed matter systems by holography, we constructed the generalized superconductors from the coupling of a scalar field to the Einstein tensor in the massive gravity and ana-

lyzed the effects of the graviton mass and Einstein tensor on the holographic superconductor models and their negative refraction. In the probe limit, we found that the critical chemical potential increases with the increase of the graviton mass or Einstein tensor coupling parameter, which supports the findings in Refs. [12,13] and indicates that the larger graviton mass or Einstein tensor parameters hinder the formation of the condensation. Interestingly, in our model we observed that the second-order transition occurs before the first-order transition to a new superconducting phase when the chemical potential increases (namely the temperature decreases), i.e, the so-called Cave of Winds, and the larger graviton mass but smaller coupling parameter make it easier for the emergence of the Cave of Winds. On the other hand, we investigated the optical properties of our generalized holographic superconductors and found the existence of the negative DepineLakhtakia index in the superconducting phase at small frequencies. We noted that the larger graviton mass but smaller Einstein tensor coupling parameter make the range of frequencies or the range of temperatures larger for which a negative DL index occurs, which means that the graviton mass and Einstein tensor have completely different effects 

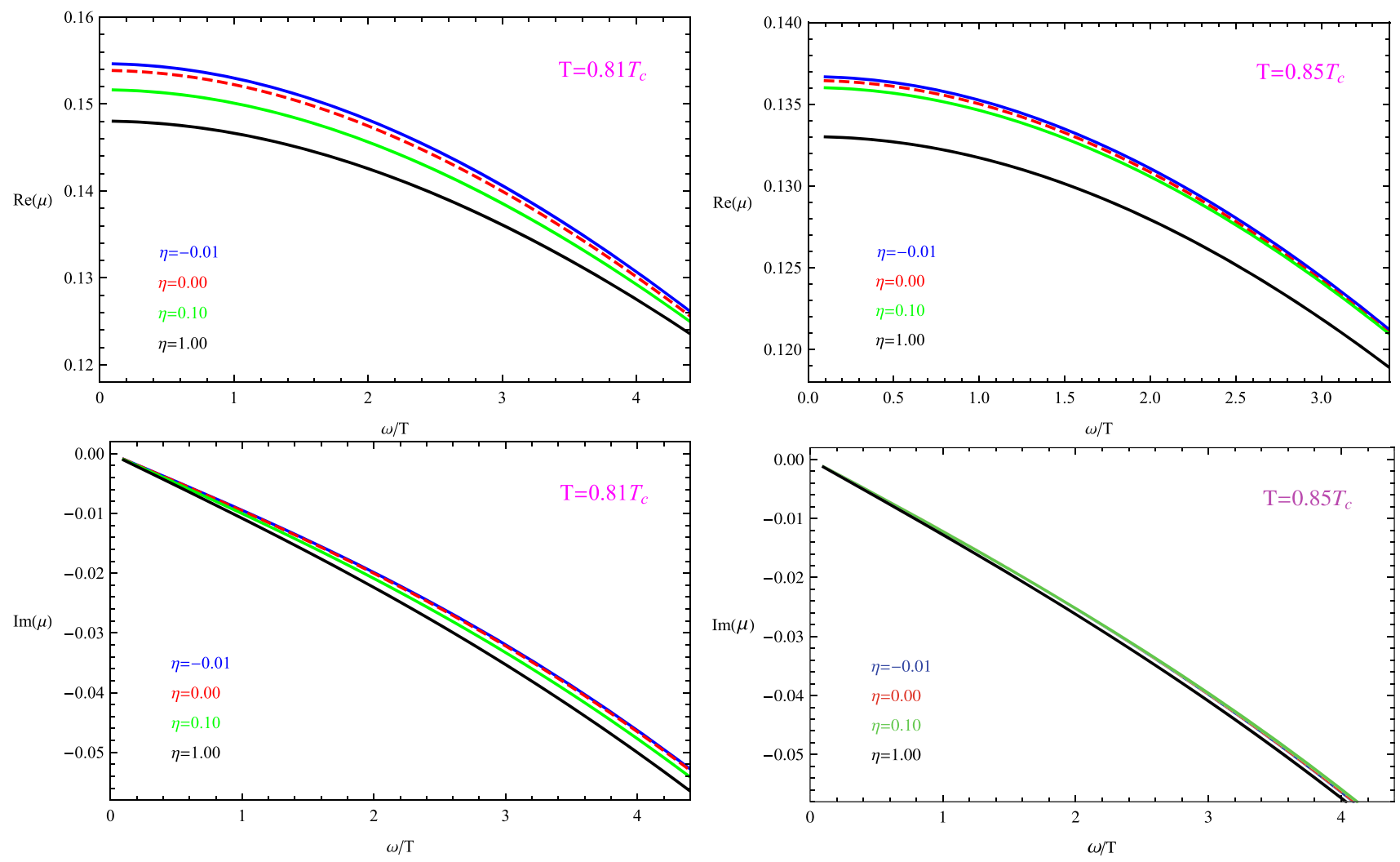

Fig. 9 Real (top) and imaginary (bottom) parts of the permeability $\mu$ as a function of $\omega / T$ with the fixed temperatures $T=0.81 T_{c}$ (left) and $T=0.85 T_{c}$ (right) for different values of the coupling parameter,

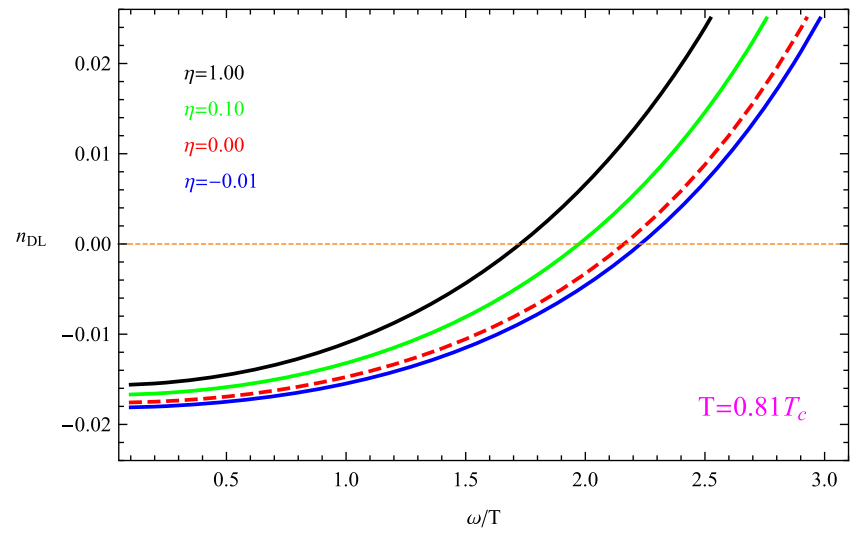

Fig. 10 Depine-Lakhtakia index $n_{D L}$ as a function of $\omega / T$ with the fixed temperatures $T=0.81 T_{c}$ (left) and $T=0.85 T_{c}$ (right) for different values of the coupling parameter, i.e., $\eta=-0.01$ (blue), 0.00

on the negative refraction. Considering the diverse effects of the doping or impurity on the refractive index of the real material, for example $\mathrm{LiNbO}_{3}[57,58]$, our result suggests that the doping or impurity will have various effects on the negative refraction in the high-temperature superconductor i.e., $\eta=-0.01$ (blue), 0.00 (red dotted), 0.10 (green) and 1.00 (black), respectively. We have set the graviton mass $m=1.0$ in the numerical computation

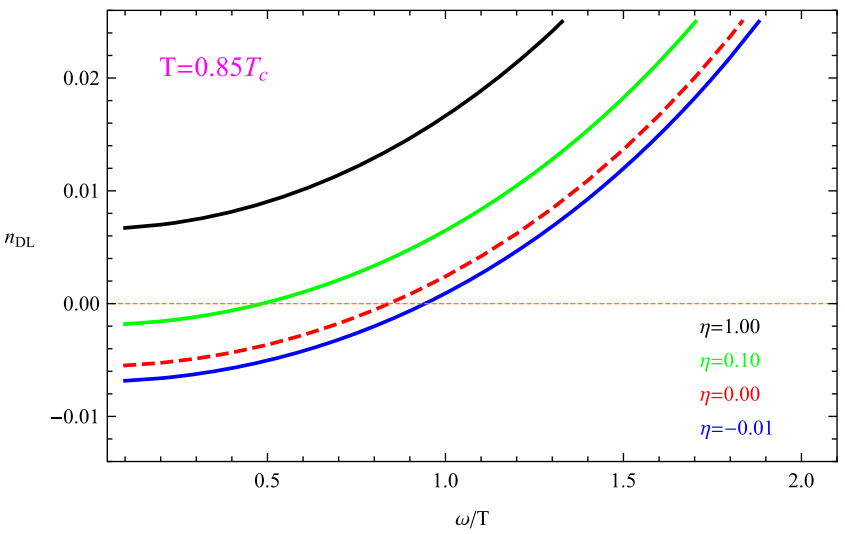

(red dotted), 0.10 (green) and 1.00 (black), respectively. We have set the graviton mass $m=1.0$ in the numerical computation

systems. Furthermore, we found that the larger graviton mass and coupling parameters both can reduce the dissipation and improve the propagation in the holographic setup. Our analysis shows that the graviton mass and the coupling of the scalar field to the Einstein tensor both can play important 

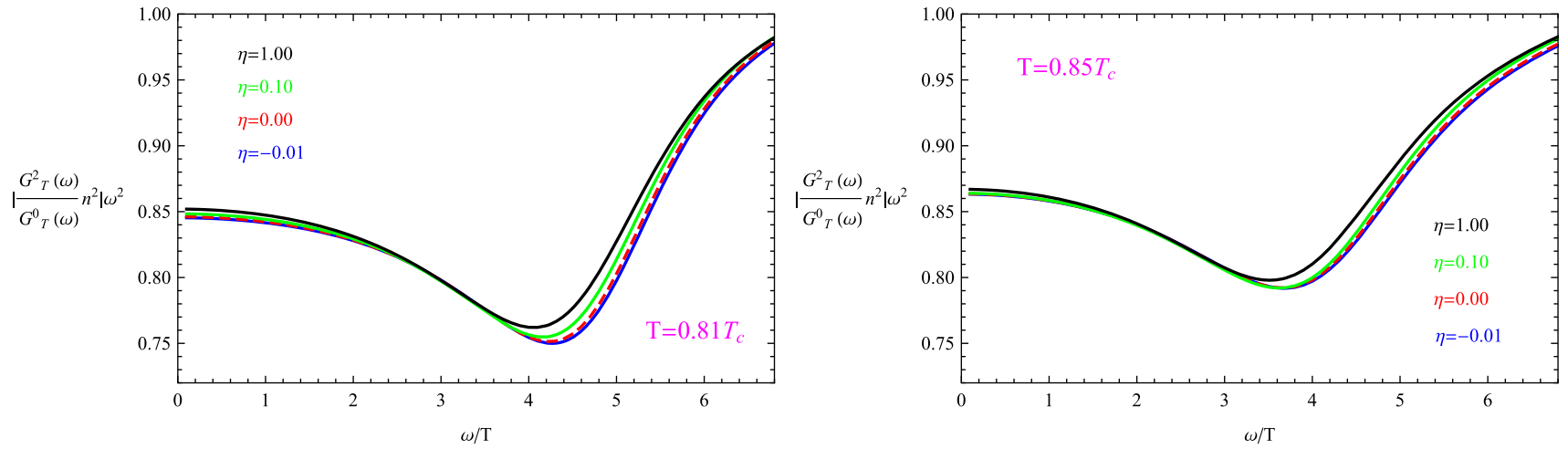

Fig. $11\left|\frac{G_{T}^{2}(\omega)}{G_{T}^{0}(\omega)} n^{2}\right| \omega^{2}$ as a function of $\omega / T$ with the fixed temperatures $T=0.81 T_{c}$ (left) and $T=0.85 T_{c}$ (right) for different values of the coupling parameter, i.e., $\eta=-0.01$ (blue), 0.00 (red dotted), 0.10 (green) and 1.00 (black), respectively. We have set the graviton mass $m=1.0$ in the numerical computation

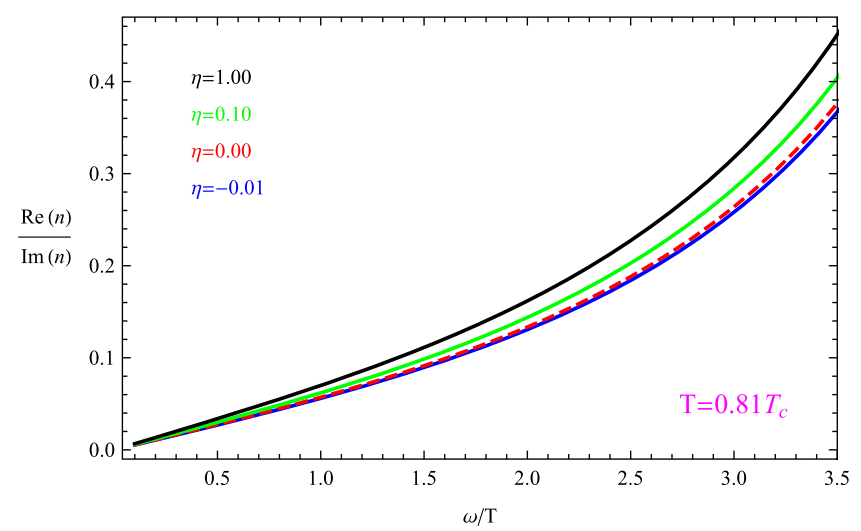

Fig. 12 Ratio $\operatorname{Re}(n) / \operatorname{Im}(n)$ as a function of $\omega / T$ with the fixed temperatures $T=0.81 T_{c}$ (left) and $T=0.85 T_{c}$ (right) for different values of the coupling parameter, i.e., $\eta=-0.01$ (blue), 0.00 (red dotted),

roles in determining the optical properties of the boundary theory.

Acknowledgements This work was supported by the National Natural Science Foundation of China under Grant nos. 11775076, 11875025, 11475061 and 11690034; Hunan Provincial Natural Science Foundation of China under Grant no. 2016JJ1012.

Data Availability Statement This manuscript has no associated data or the data will not be deposited. [Authors' comment: This is a theoretical study and no experimental data has been listed.]

Open Access This article is distributed under the terms of the Creative Commons Attribution 4.0 International License (http://creativecomm ons.org/licenses/by/4.0/), which permits unrestricted use, distribution, and reproduction in any medium, provided you give appropriate credit to the original author(s) and the source, provide a link to the Creative Commons license, and indicate if changes were made. Funded by SCOAP ${ }^{3}$.

\section{References}

1. J. Maldacena, Adv. Theor. Math. Phys. 2, 231 (1998)

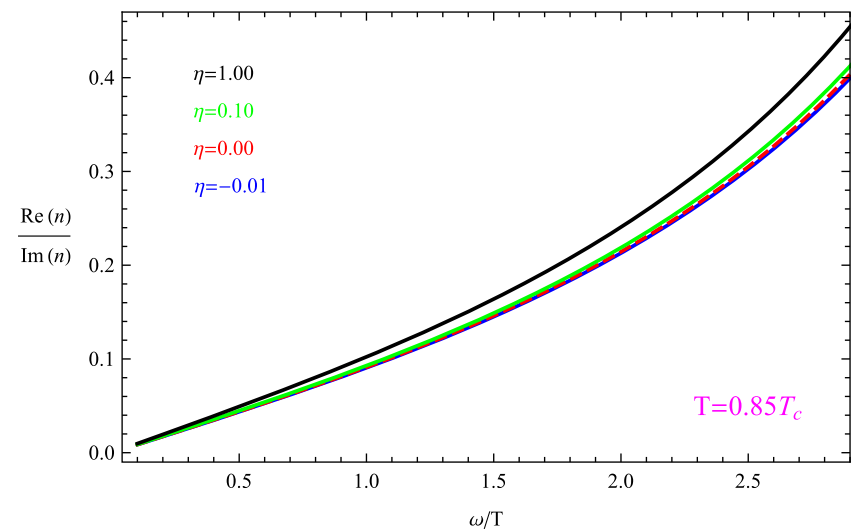

0.10 (green) and 1.00 (black), respectively. We have set the graviton mass $m=1.0$ in the numerical computation

2. J. Maldacena, Int. J. Theor. Phys. 38, 1113 (1999)

3. E. Witten, Adv. Theor. Math. Phys. 2, 253 (1998)

4. S.S. Gubser, I.R. Klebanov, A.M. Polyakov, Phys. Lett. B 428, 105 (1998)

5. S.A. Hartnoll, C.P. Herzog, G.T. Horowitz, Phys. Rev. Lett. 101, 031601 (2008)

6. J. Zaanen, Y.W. Sun, Y. Liu, K. Schalm, Holographic Duality in Condensed Matter Physics (Cambridge University Press, Cambridge, 2015)

7. S.A. Hartnoll, Class. Quant. Grav. 26, 224002 (2009)

8. C.P. Herzog, J. Phys. A 42, 343001 (2009)

9. G.T. Horowitz, Lect. Notes Phys. 828, 313 (2011). arXiv: 1002.1722 [hep-th]

10. R.G. Cai, L. Li, L.F. Li, R.Q. Yang, Sci. China Phys. Mech. Astron. 58, 060401 (2015). arXiv:1502.00437 [hep-th]

11. T. Ishii, S.J. Sin, J. High Energy Phys. 1304, 128 (2013). arXiv:1211.1798 [hep-th]

12. Y.P. Hu, H.F. Li, H.B. Zeng, H.Q. Zhang, Phys. Rev. D 93, 104009 (2016)

13. X.M. Kuang, E. Papantonopoulos, J. High Energy Phys. 1608, 161 (2016). arXiv:1607.04928 [hep-th]

14. G. Filios, P.A. González, X.M. Kuang, E. Papantonopoulos, Y. Vásquez. arXiv:1808.07766 [hep-th]

15. D. Wang, M.M. Sun, Q.Y. Pan, J.L. Jing, Phys. Lett. B 785, 362 (2018) 
16. H.B. Zeng, H.Q. Zhang, Nucl. Phys. B 897, 276 (2015). arXiv:1411.3955 [hep-th]

17. L.Q. Fang, X.M. Kuang, B. Wang, J.P. Wu, J. High Energy Phys. 1511, 134 (2015)

18. W.J. Jiang, H.S. Liu, H. Lu, C.N. Pope, J. High Energy Phys. 1707, 084 (2017). arXiv:1703.00922 [hep-th]

19. M. Baggioli, W.J. Li, J. High Energy Phys. 1707, 055 (2017). arXiv:1705.01766 [hep-th]

20. V.G. Veselago, Sov. Phys. Usp. 10, 509 (1968)

21. D.R. Smith, W.J. Padilla, J. Willie, D.C. Vier, S.C. Nemat-Nasser, S. Schultz, Phys. Rev. Lett. 84, 4184 (2000)

22. J.B. Pendry, Phys. Rev. Lett. 85, 3966 (2000)

23. S.A. Ramakrishna, Rep. Prog. Phys. 68, 449 (2005)

24. S.M. Anlage, J. Opt. 13, 024001 (2011). arXiv:1004.3226 [condmat]

25. P. Jung, A.V. Ustinov, S.M. Anlage, Supercond. Sci. Technol. 27, 073001 (2014). arXiv:1403.6514 [cond-mat]

26. X. Gao, H.B. Zhang, J. High Energy Phys. 1008, 075 (2010). arXiv: 1008.0720 [hep-th]

27. A. Amariti, D. Forcella, A. Mariotti, M. Siani, J. High Energy Phys. 1110, 104 (2011). arXiv:1107.1242 [hep-th]

28. S. Mahapatra, P. Phukon, T. Sarkar, J. High Energy Phys. 1401, 135 (2014)

29. S. Franco, A.M. Garcia-Garcia, D. Rodriguez-Gomez, Phys. Rev. D 81, 041901(R) (2010)

30. S. Franco, A.M. Garcia-Garcia, D. Rodriguez-Gomez, J. High Energy Phys. 1004, 092 (2010)

31. Q.Y. Pan, B. Wang, Phys. Lett. B 693, 159 (2010). arXiv: 1005.4743 [hep-th]

32. R.A. Depine, A. Lakhtakia, Microw. Opt. Tech. Lett. 41, 315 (2004). arXiv:physics/0311029

33. J. Cheng, Q.Y. Pan, H.W. Yu, J.L. Jing, Eur. Phys. J. C 78, 239 (2018). arXiv:1803.08204 [hep-th]

34. A. Amariti, D. Forcella, A. Mariotti, G. Policastro, J. High Energy Phys. 1104, 036 (2011). arXiv:1006.5714 [hep-th]

35. X.H. Ge, K. Jo, S.J. Sin, J. High Energy Phys. 1103, 104 (2011). arXiv: 1012.2515 [hep-th]

36. J. Liu, M.J. Luo, Q. Wang, H.J. Xu, Phys. Rev. D 84, 125027 (2011)
37. A. Amariti, D. Forcella, A. Mariotti, J. High Energy Phys. 1301, 105 (2013). arXiv:1107.1240 [hep-th]

38. B.F. Jiang, D.F. Hou, J.R. Li, Y.J. Gao, Phys. Rev. D 88, 045014 (2013)

39. P. Phukon, T. Sarkar, J. High Energy Phys. 1309, 102 (2013)

40. A. Dey, S. Mahapatra, T. Sarkar, J. High Energy Phys. 1406, 147 (2014). arXiv:1404.2190 [hep-th]

41. D. Forcella, A. Mezzalira, D. Musso, J. High Energy Phys. 1411, 153 (2014)

42. S. Mahapatra, J. High Energy Phys. 1501, 148 (2015)

43. B.F. Jiang, D.F. Hou, J.R. Li, Phys. Rev. D 94, 074026 (2016)

44. M.K. Zangeneh, S.S. Hashemi, A. Dehyadegari, A. Sheykhi, B. Wang, Phys. Lett. B 785, 238 (2018). arXiv:1710.10162 [hep-th]

45. D. Arean, P. Basu, C. Krishnan, J. High Energy Phys. 1010, 006 (2010)

46. Y.B. Wu, J.W. Lu, W.X. Zhang, C.Y. Zhang, J.B. Lu, F. Yu, Phys. Rev. D 90, 126006 (2014). arXiv: 1410.5243 [hep-th]

47. Y.B. Wu, J.W. Lu, C.Y. Zhang, N. Zhang, X. Zhang, Z.Q. Yang, S.Y. Wu, Phys. Lett. B 741, 138 (2015). arXiv:1412.3689 [hep-th]

48. S.C. Liu, Q.Y. Pan, J.L. Jing, Phys. Lett. B 765, 91 (2017). arXiv: 1610.02549 [hep-th]

49. D. Vegh. arXiv:1301.0537 [hep-th]

50. R.G. Cai, Y.P. Hu, Q.Y. Pan, Y.L. Zhang, Phys. Rev. D 91, 024032 (2015)

51. S.A. Hartnoll, C.P. Herzog, G.T. Horowitz, J. High Energy Phys. 0812, 015 (2008)

52. L.D. Landau, E.M. Lifshitz, Electrodynamics of Continous Media (Pergamon Press, Oxford, 1984)

53. M. Dressel, G. Gruner, Electrodynamics of Solids (Cambridge University Press, Cambridge, 2002)

54. D.T. Son, A.O. Starinets, J. High Energy Phys. 0209, 042 (2002). arXiv:hep-th/0205051

55. M.W. McCall, A. Lakhtakia, W.S. Weiglhofer, Eur. J. Phys. 23, 353 (2002)

56. V.A. Markel, Phys. Rev. E 78, 026608 (2008)

57. M. Minakata, S. Saito, M. Shibata, S. Miyazawa, J. Appl. Phys. 49, 4677 (1978)

58. L. Pálfalvi, J. Hebling, J. Kuhl, Á. Péter, K. Polgár, J. Appl. Phys. 97, $123505(2005)$ 